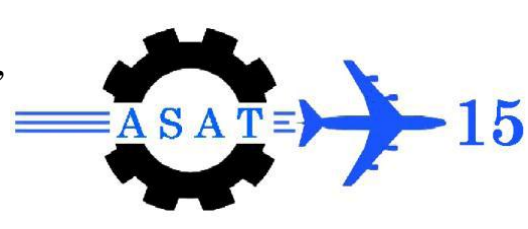

\title{
Performance Evaluation of Serially Concatenated LDPC and Reed-Solomon Codes for OFDM Systems
}

\author{
A. Mahran*, and M. Karem ${ }^{\dagger}$
}

\begin{abstract}
Both high quality and high-bit-rate transmission' are critical demands for recent mobile communication system. In this multipath environment OFDM has been proven as an attractive technique. As errors can still occur in the received data; channel coding is a key component is these systems. Since its reintroduction, Low-Density Parity-Check (LDPC) codes have gained much attention in the field of coding theory. Several researches have been carried out regarding concatenating the LDPC with other codes. This work introduces the use of serial concatenation of Reed-Solomon and LDPC. Two schemes of such concatenation will be investigated. The first one uses hard decision decoding of Reed-Solomon code while the other one will get use of the reliability output of the LDPC decoder to be fed to Chase-II softdecision algorithm to decode the outer Reed-Solomon code.
\end{abstract}

Keywords: Channel Coding, LDPC, Reed-Solomon, OFDM.

\section{Introduction}

Higher rates communication is an everlasting demand for today's civilization. Channel coding is one major player to achieve this goal. Many milestones have been seen in this road which boarded by Claude Shannon theory. One interesting milestone appeared in this road is the Low-Density Parity-Check (LDPC) codes which have attracted much attention in the field of coding theory [1], [2]. It has a performance very close to the Shannon limit with practical decoding complexity like Turbo codes [3], [4]. Meanwhile, OFDM has been proven a good technique for the high-bit-rate data transmission in a multipath environment that causes inter symbol interference (ISI). The wide signal bandwidth is divided into many narrow subbands that are transmitted in parallel subchannel. Moreover, a guard interval is added to mitigate the ISI such that when each subchannel is taken into consideration, it apparently undergoes flat fading [5]. In spite the fact that OFDM randomizes the burst errors caused by fading, many OFDM symbols can be distorted in case of deep fades. Increasing the guard interval and the number of subcarriers are known as a typical solution to tackle these problems.

The significant coding gain improvement of Coded OFDM over OFDM has encouraged many researchers to study the performance of Coded-OFDM over different channels. LDPC was one channel code that shows better performance for OFDM systems [6]-[9]. In [6], the performance of LDPC-OFDM system over AWGN and known frequency selective fading channel is investigated while in [7] an investigation of LDPC-OFDM for fixed wireless

\footnotetext{
*Egyptian Armed Forces, Egypt, a.mahran@ieee.org

${ }^{\dagger}$ Egyptian Armed Forces, Egypt, $\underline{\text { m.karemm@hotmail.com }}$
} 
application is presented. The performance of high data rate multiband OFDM systems over 1 Gbps using different forms of LDPC code is evaluated in [8]. In [9] flexible LDPC code is designed and optimized for OFDM system in aeronautical channel.

Motivated by the success of LDPC over OFDM system; a new research trend has been started. It focuses on concatenating LDPC codes with an outer code. Reed-Solomon code is a strong candidate for concatenation. This is because its superior performance to correct burst errors which are quite often to occur during deep fades. There have been many forms of this concatenation between Reed-Solomon and LDPC code. In [10] an algebraic method for constructing regular LDPC codes based on Reed-Solomon codes was used. In [11], a quasicyclic LDPC (QCLDPC) codes based on a two-dimensional Reed-Solomon codes gives good performance with the sum-product algorithm (SPA). The work in [12], [13] develop a parallel concatenation of Reed-Solomon and LDPC with size matched to 10GBASE-T Ethernet. This scheme was able to lower the error floor to below $10^{-14}$.

This paper introduces a serial concatenation of Reed-Solomon codes with two decoding approaches. The first decoding process will use the hard output of the LDPC code and decoding of Reed-Solomon will be carried out using Berlekamp-Massey algorithm [14]. The second approach will get use reliability measure of the output from LDPC decoder and then uses Chase-II soft decision algorithm [15] to decode the binary equivalent Reed-Solomon code.

The paper is organized as follows. The system model will be illustrated in section 2 . In section 3 Reed-Solomon code are will be explained and its hard decision decoding algorithm. Chase-II algorithm for binary equivalent Reed-Solomon will be adapted in section 4. Section 5 will show the details of the sum-product algorithm for decoding LDPC code. Serial concatenation between Reed-Solomon and LDPC will be introduced in section 6. Simulations results which validate the proposed approach are shown in the section 7 . Conclusions are figured out in section 8 .

\section{Coded OFDM System Model}

In this section the system model and design parameters for the proposed COFDM scheme are presented. In Fig. 1 a block diagram of a COFDM transceiver is shown. The total available channel bandwidth $B$ is divided into a number of subcarriers $N$, each having a bandwidth $B / N$.

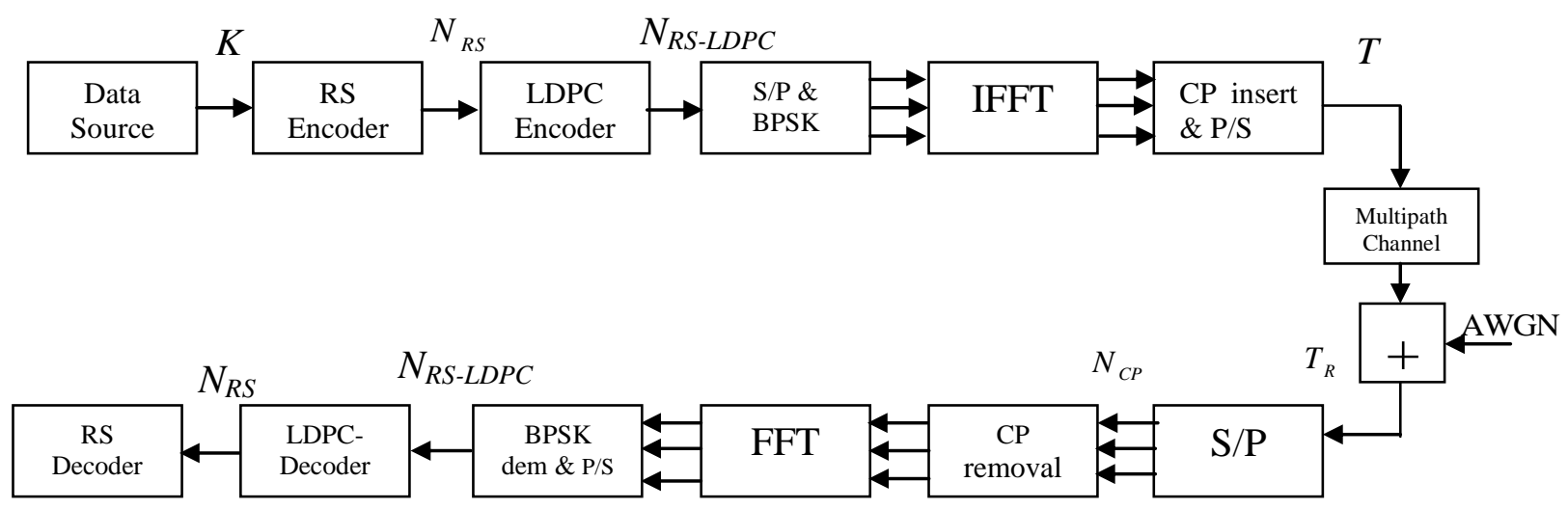

Fig. 1 Block diagram of the coded-OFDM transceiver.

The data of a specific user, after the application of channel coding, is used to modulate the $N$ subcarriers by using binary PSK (BPSK). After the subcarrier modulation, the transmit signal is produced by first applying inverse fast Fourier transform (IFFT) to the modulated subcarriers and extending the IFFT output by a precursor signal of $T$ samples, which equals 
the last $N_{G I}$ samples of the IFFT output and is termed cyclic prefix (CP). The resulting signal constitutes an OFDM symbol and is transmitted through the wireless multipath channel. At the receiver side, the first $N_{G I}$ samples of the received signal $T_{R}$ are discarded, an operation denoted CP removal. Then FFT is applied to the remaining signal. Then data demodulation at each subcarrier takes place, and fed into the channel decoder.

The pilot symbols are inserted into the modulated symbols transmitted over $N_{C}$ carriers in parallel. The multicarrier modulation is implemented using the inverse fast Fourier transform (IFFT). The baseband signal at the output of the IFFT block can be expressed as:

$$
x_{i}(n)=\left(1 / \sqrt{N_{C}}\right) \sum_{k=0}^{N_{C}-1} X_{i}(k) e^{j 2 \pi k n / N_{C}}
$$

for $n=0,1, \ldots, N_{C}-1$ where $n$ represents the time index and $k$ is the subcarrier index. A guard interval is inserted between consecutive OFDM blocks to prevent ISI and is chosen to be larger than the expected delay spread. By inserting the cyclically extended part of OFDM block into the guard interval, it helps to maintain the orthogonality between different subcarriers and eliminates intercarrier interference. The transmitted OFDM symbols after inserting the guard interval is given by:

$$
x_{i}^{t}(n)=\left\{\begin{array}{cc}
x_{i}(N+n), & n=-N_{g},-N_{g}+1, \ldots,-1 \\
x_{i}(n) & n=0,1, \ldots, N-1
\end{array}\right.
$$

where $N$ is the number of symbols in the block of OFDM, $N_{g}$ is the number of symbols in the guard interval. The total length of an OFDM block is $\left(N_{g}+N_{C}\right)$. The channel model used in this system is a tapped delay line model with time-varying coefficients and fixed tap spacing $T_{s}$, where $T_{s}$ is the time sampling. The channel is assumed to be a wide-sense-stationary uncorrelated scattering (WSSUS) Rayleigh fading channel $h(n)$. After the transmitted signal $x_{i}^{t}(n)$ passes through the channel, the received signal can be written as:

$$
y_{i}^{t}(n)=x_{i}^{t}(n) \otimes h_{i}(n)+w(n)
$$

where $\otimes$ is used for circular convolution, and $w(n)$ is a zero-mean AWGN with variance $\sigma_{w}^{2}$. After discarding the guard interval symbols, the received signal in the frequency domain is obtained by using FFT and can be written as:

$$
\begin{aligned}
Y_{i}(k) & =1 / \sqrt{\left(N_{C}\right)} \sum_{n=0}^{N_{C}-1} y_{i}(n) e^{-j 2 \pi n k / N_{C}} \\
& =X_{i}(k) H_{i}(k)+W_{i}(k)
\end{aligned}
$$

where $y_{i}(n)$ denotes the received signal after removing the guard interval, $H_{i}(k)$ is DFT of $h_{i}(n)$, and $W_{i}(k)$ is the DFT of $w(n)$. The above equations can be also expressed in a matrixvector model, which will facilitate later analysis. First, let some basic vector will be defined. Note that we omit subscript $i$ for notational simplicity. The transmitted signal in frequency domain and time domain can be written respectively as:

$$
\begin{gathered}
\mathbf{X}=\left[\begin{array}{llll}
X(0) & X(1) & \ldots & X\left(N_{C}-1\right)
\end{array}\right]^{\mathrm{T}} \\
\mathbf{x}=\left[\begin{array}{llll}
x(0) & x(1) & \ldots & x\left(N_{C}-1\right)
\end{array}\right]^{\mathrm{T}}
\end{gathered}
$$


where [.] $]^{\mathrm{T}}$ denotes transpose. Normally, $\mathbf{X}$ is used to express frequency domain signal, $\mathbf{x}$ is for time domain signal. Their relationship can be written as:

$$
\mathbf{x}=\mathbf{F}^{H} \mathbf{X}
$$

where $\mathbf{F}$ denotes the FFT transform matrix, and $\mathbf{F}=\left(1 / N_{C}\right)^{1 / 2} \exp \left(-j 2 \pi m n / N_{C}\right)$. where $n$ is number of column, and $m$ is number of row. Similarly, the received signals can be expressed by:

$$
\begin{aligned}
& \mathbf{Y}=\left[\begin{array}{llll}
Y(0) & Y(1) & \ldots & Y\left(N_{C}-1\right)
\end{array}\right]^{\mathrm{T}} \\
& \mathbf{y}=\left[\begin{array}{llll}
\mathrm{y}(0) \mathrm{y}(1) & \ldots \mathrm{y}\left(N_{C}-1\right)
\end{array}\right]^{\mathrm{T}}
\end{aligned}
$$

and their relationship is:

$$
\mathbf{y}=\mathbf{F}^{H} \mathbf{Y}
$$

if the channel frequency response is denoted by:

$$
\begin{gathered}
\mathbf{H}=\left[\begin{array}{llll}
H(0) & H(1) & \ldots & H\left(N_{C}-1\right)
\end{array}\right]^{\mathrm{T}} \\
\mathbf{h}=\left[\begin{array}{llll}
h(0) & h(1) & \ldots & h\left(N_{C}-1\right)
\end{array}\right]^{\mathrm{T}}
\end{gathered}
$$

Then (4) can be rewritten as:

$$
\mathbf{Y}=\mathbf{H} \square \mathbf{X}+\mathbf{W}
$$

where $\square$ denotes the Hadamard product of the vector $\mathbf{X}$ and $\mathbf{H}$.

\section{Reed Solomon Code}

Reed-Solomon codes have many attractive features. They are Maximum distance separable (MDS) codes which means that they have the lowest number of redundancy for same coderate amongst all other block codes. Nonetheless, Reed-Solomon codes can correct burst errors of length determined by the code parameters. For these reasons, Reed-Solomon codes are widely used in the applications of communication and data storages. RS codes can be denoted by $\left(N_{R S}, K\right)$, where $N_{R S}$ and $K$ represent the length of a block and the length of the information symbols, respectively. It can correct up to $t=\left\lfloor\left(N_{R S}-K\right) / 2\right\rfloor$ symbols [14].

Reed-Solomon codes are the special subclass of $q^{m}$-ary BCH codes with $s=1$. The generator polynomial $g(x)$ of $t$-error-correcting RS codes has roots that are $\left(d_{m i n}-1\right)$ consecutive powers of a primitive element $\alpha$, and is represented by

$$
g(X)=\prod_{i=1}^{2 t}\left(x-\alpha^{i}\right)
$$

The parameters of the RS codes are shown in Table 1.

Table 1. Parameters of RS Codes

\begin{tabular}{l|l}
\hline \hline Block length & $\mathrm{n}=\mathrm{q}^{\mathrm{m}}-1$ \\
\hline Number of parity check digits & $\mathrm{n}-\mathrm{k}=2 \mathrm{t}$ \\
\hline Minimum distance & $\mathrm{d}_{\min }=2 \mathrm{t}+1$ \\
\hline \hline
\end{tabular}




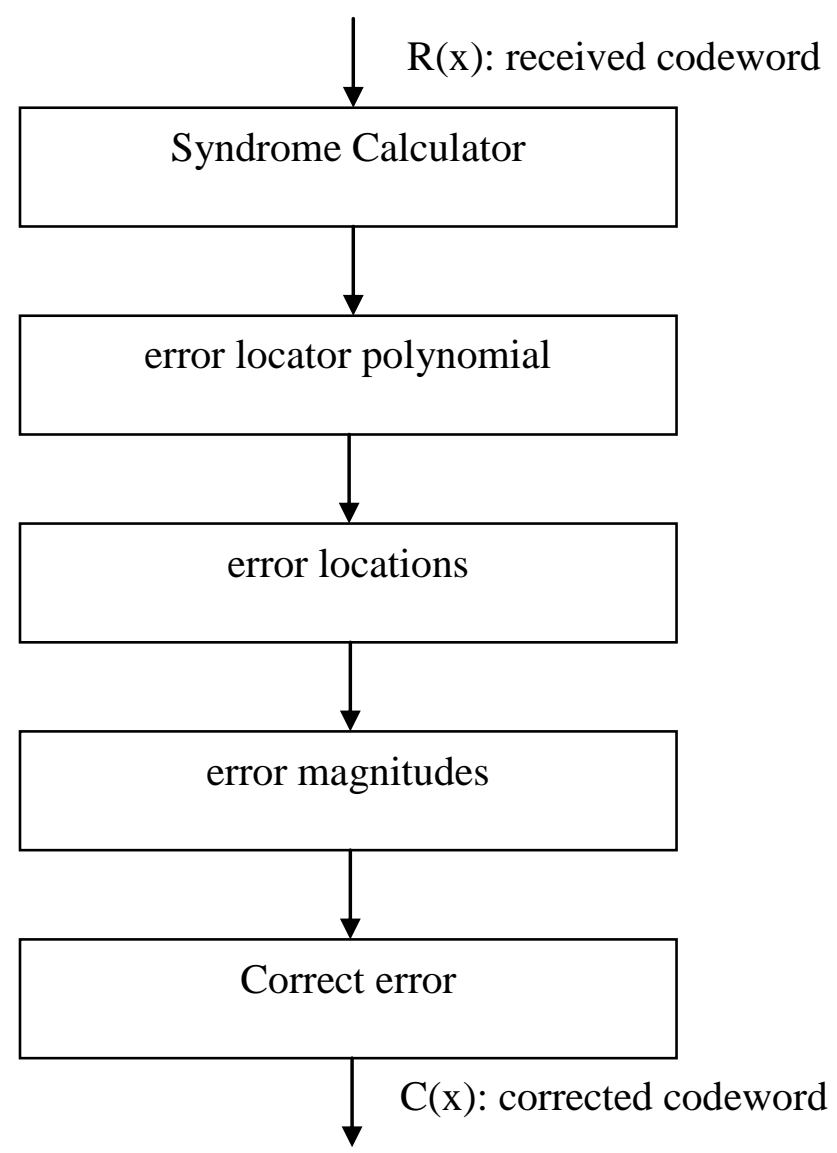

Fig. 2 Reed Solomon decoder architecture

The received polynomial $R(x)$ can be decomposed as:

$$
\mathrm{R}(\mathrm{x})=\mathrm{C}(\mathrm{x})+\mathrm{E}(\mathrm{x})
$$

Let $E(x)=E_{0}+E_{1} x+\ldots+E_{n-1} x^{n-1}$ be the expansion of the error polynomial. Assume that there are at most $s$ errors. Let $j_{1}, j_{2}, \ldots, j_{s}$ denote the positions of the errors. The error locations can be defined as:

The error Location $X_{i}$ can be defined as $X_{i}=\alpha^{j_{i}}$ and the error magnitude $Y_{i}$ is defined as $E_{j_{i}}$ where both $X_{i}$ and $Y_{i}$ are elements of $\operatorname{GF}(q)$. The first step in decoding a received message $R(x)$ is to compute its syndromes. The syndromes are defined as:

The syndrome $s_{l}$, for $l \leq l \leq s$, of the received message is the polynomial $R(x)$ evaluated at $\alpha^{l}$, i.e., $s_{l}=R(\alpha)$.

Since $C\left(\alpha^{l}\right)=0$, we have $s_{l}=R\left(\alpha^{l}\right)=E\left(\alpha^{l}\right)$ for $1 \leq l \leq 2 s$. Thus,

$$
s_{l}=E\left(\alpha^{l}\right)=\sum_{i=1}^{s} Y_{i} \alpha^{l j_{i}}=\sum_{i=1}^{s} Y_{i} X_{i}^{l}
$$

and the syndrome polynomial can be defined as:

$$
s(z)=\sum_{i=1}^{\infty} s_{i} z^{i}
$$

Having computed the syndromes, the error locator and error magnitude polynomials have to be solved. The error locator polynomial $\sigma(z)$ can be defined as:

$$
\sigma(z)=\prod_{i=1}^{s}\left(1-X_{i} z\right)
$$

While the error magnitude polynomial $\omega(z)$ can be defined as: 


$$
\omega(z)=\sigma(z)+\sum_{i=1}^{s} z X_{i} Y_{i} \prod_{\substack{j=1 \\ j \neq i}}^{s}\left(1-X_{j} z\right)
$$

The equation which relates the three polynomials $s(z), \sigma(z)$, and $\omega(z)$ is called the key equation:

$$
(1+s(z)) \sigma(z)=\omega(z) \bmod z^{2 s+1}
$$

Two widely used methods to compute $\sigma(z)$ and $\omega(z)$ are; the extended Euclidean algorithm and the Berlekamp-Massey algorithm. Having solved for $\sigma(z)$, the Chein search algorithm is used to compute the error locations $X_{i}$. Finally, Forney's formula gives the error magnitudes as follows:

$$
\omega\left(X_{l}^{-1}\right)=Y_{l} \prod_{\substack{j=1 \\ j \neq l}}^{s}\left(1-X_{j} X_{l}^{-1}\right)
$$

As the solution for the error polynomial $E(x)$ has been found, the codeword $C(x)$ can be computed as: $C(x)=R(x)-E(x)$, and the decoding is complete.

\section{Soft-Decision Decoding of Reed-Solomon Using Chase-II Algorithm}

Although Reed-Solomon codes are mainly non-binary codes; they can be used for binary data since every element in $\mathrm{GF}\left(2^{m}\right)$ can be represented uniquely by a binary m-tuple, called an $\mathrm{m}$ bit symbol. Suppose a Reed-Solomon $\left(N_{R S}, K\right)$ code with symbols from $\mathrm{GF}\left(2^{m}\right)$ is used for encoding binary data. A message of $\mathrm{Km}$ bits is first divided into $K$ groups each of $\mathrm{m}$-bits. Each $\mathrm{m}$-bits is regarded as a symbol in $\mathrm{GF}\left(2^{m}\right)$. The $K$ symbols are then encoded into Nsymbols codeword base on the Reed-Solomon encoding algorithm. By this way we have got a binary codeword encoded by what is called the binary equivalent Reed-Solomon code. This allows the use of soft decision decoding algorithms used for binary $\mathrm{BCH}$ codes to be used for soft-decision decoding of Reed-Solomon codes. In this work the sub-optimal Maximum Likelihood decoding (MLD) algorithm, Chase-II, will be used for Reed-Solomon decoding using the soft-output from the inner LDPC decoder.

Consider the transmission of codeword vector $X$ encoded using a linear block code $C$ with parameters $\left(n, k, d_{\text {min }}\right)$ where $n$ is the code length, $k$ is the number of information bits, and $d_{\text {min }}$ is the minimum distance, on a Gaussian channel using BPSK, i.e. $0 \rightarrow-1$ and $1 \rightarrow+1$. At the receiver the received sequence $Y=\left(y_{1}, \ldots, y_{l}, \ldots, y_{n}\right)$ at the output of the Gaussian channel for a transmitted codeword $X=\left(x_{1}, \ldots, x_{l}, \ldots, x_{n}\right)$ is given by

$$
Y=X+G
$$

where components $g_{l}$ of $\boldsymbol{G}=\left(\boldsymbol{g}_{l}, \ldots, \boldsymbol{g}_{l}, \ldots, \boldsymbol{g}_{n}\right)$ are additive white Gaussian noise (AWGN) samples of standard deviation $\sigma$. For binary symmetric channel elements of $Y$ are quantized to 0 and 1 . This is equivalent to deciding which one of the two logical values was transmitted. In other words, no information is passed about the reliability of the hard decision. Better results can be expected when the quantized analogue received signal is passed directly to the decoder. In that case, the demodulator will generate the reliability sequence $r=\left(r_{l}, \ldots, r_{l}, \ldots\right.$, $r_{n}$ ) from $Y$.

The reliability of the component $y_{j}$ can be defined using the log-likelihood ratio (LLR) of decision $y_{j}$

$$
\Lambda\left(y_{j}\right)=r_{j}=\ln \left(\frac{\operatorname{Pr}\left\{x_{j}=+1 \mid y_{j}\right\}}{\operatorname{Pr}\left\{x_{j}=-1 \mid y_{j}\right\}}\right)=\left(\frac{2}{\sigma^{2}}\right) y_{j}
$$

If we consider a stationary channel, we can normalize the LLR with respect to constant $2 / \sigma^{2}$, and the reliability of $y_{j}$ is then given by $\left|y_{j}\right|$. To find the optimum decision $D=\left(d_{l}, \ldots, d_{l}, \ldots\right.$, $d_{n}$ ) corresponding to the transmitted codeword $X$, one has to search through all the codewords 
of a given block code. The Maximum Likelihood (ML) codeword is the closest to the received sequence $Y$ as follows:

$$
D=\min \left[\sum_{j=1}^{n}\left|r_{j}\right| \cdot\left(Y^{H} \oplus C^{i}\right)\right]
$$

where $C^{i}=\left(c_{1}^{i}, \ldots, c_{l}^{i}, \ldots, c_{n}^{i}\right)$ is the $i^{\text {th }}$ codeword of $C$ and $Y^{H}=\left(y_{1}{ }^{H}, y_{2}{ }^{H} \ldots, y_{n}{ }^{H}\right)$ is the hard decision decoded sequence of $R$ and $x_{j}$ defined as:

$$
y_{j}^{H}= \begin{cases}0 & r_{\mathrm{j}}<0 \\ 1 & r_{j} \geq 0\end{cases}
$$

For an $\left(n, k, d_{\text {min }}\right)$ block code, MLD searches all the $2^{K}$ codewords to find the ML codeword, $D$. Altho0ugh, optimum performance can be achieved by using MLD, this type of decoding algorithm is associated with a prohibitive complexity increasing exponentially with $k$. Chase [15] presented a sub-optimum decoding algorithm, which uses channel reliability information to limit the associated complexity. This algorithm generates a set of several candidate codewords by using hard-decision decoding and chooses the most likely one among them. The basic procedure of the Chase algorithm is subsequently explained:

1. Make hard decision on each symbol in the received sequence to produce vector $Y^{H}$.

2. Sort the received bits in an ascending order according to the their reliabilities.

3. Determine the positions of the $P=\left\lfloor d_{\text {min }} / 2\right\rfloor$ Least Reliable Bits using reliability.

4. Deliberately generate exhaustive patterns of errors, $E$, as all possible combinations of 1 's and 0 's in the positions of LRBs.

5. Form test sequences $Z=E+Y^{H}$ and algebraically decode each sequence created in this manner.

6. Compute the correlation metric $D$ for each of the codewords in $Z$ to the received sequence, and then choose the codeword with the minimum distance.

The Chase algorithm [15] can be applied with a different number of test patterns. The number of test patterns considered are $\left(\begin{array}{c}n \\ \left\lfloor d_{\min } / 2\right\rfloor\end{array}\right)$ for the Chase-I algorithm, $2^{\left\lfloor d_{\min } / 2\right\rfloor}$ for the Chase-II algorithm, and $\left\lfloor\left(d_{\min } / 2\right)+1\right\rfloor$ for the Chase-III algorithm. Due to a large number of error patterns to be tested, Chase- 1 algorithm is of little interest in practice. In addition, Chase-III algorithm improvement at practical bit error rates (BER's) is not significant. It is of interest only for large minimum distance codes since the number of test patterns grows only linearly with the minimum distance of the code. The Chase-II algorithm meets most of the requirements of Reed-Solomon since it offers a good tradeoff between performance and complexity.

\section{Low Density Parity Check Code}

LDPC codes are linear block codes defined by a very sparse parity-check matrix $\mathbf{H}_{L D P C}$. A code with rate $R=K / N$ is defined by the number of input bits $K$ in a block and the number of output bits $N$. Matrix $\mathbf{H}_{L D P C}$ is required to be full rank, with dimensions $M \times N$, where $M=N-K$. Regular LDPC codes are defined by a constant row weight of $w_{r}$ and a column weight of $w_{c}$, where $w_{c} \square M$ and $w_{r}=w_{c} N / M$ [10]. Therefore, $\mathbf{H}_{L D P C}$ has a small number 
(density) of ones, giving the code its name. If the number of one's per column or row is not constant, then the code is an irregular code [11]. In this paper, we consider regular LDPC codes. In order to avoid low-weight codeword we ensure that no two columns in the $\mathbf{H}_{L D P C}$ matrix overlap in more than one non-zero bit position.

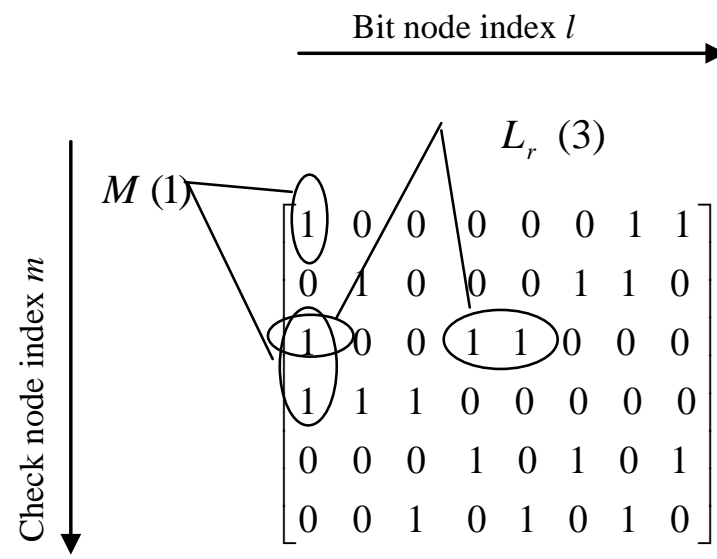

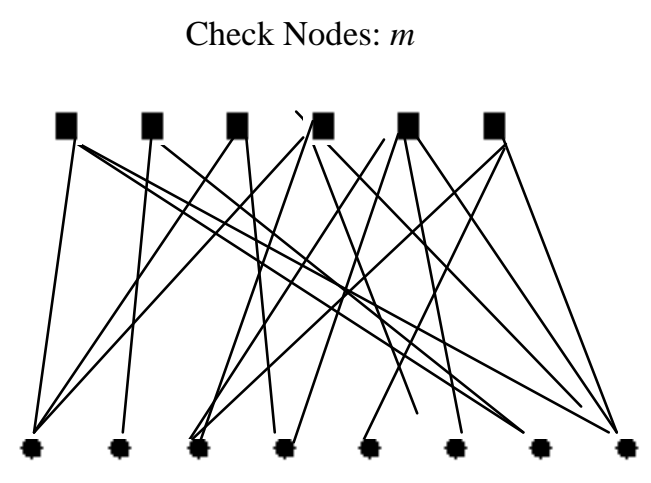

Bit Nodes: $l$

Fig. 3 Relation between the check nodes and bit nodes

The encoding of LDPC codes is the same as that of common block codes. But it has a special decoding method, an iterative probability algorithm known as the sum-product or belief propagation. At each iteration of the decoding, each bit node gets probability message from all the check nodes connected to it, and sends messages back to these check nodes after processing, The similar procedure is applied to each check node, which will receive messages from the connected bit nodes and transfer the processed messages back to these bit nodes.

In Figure 3, $M(l)$ denotes the set of check nodes that are connected to the bit node $l$, i.e., positions of " 1 "s in the $l^{\text {th }}$ column of the parity-check matrix. $L_{r}(m)$ denotes the set of bits that participates in the $m^{\text {th }}$ parity-check equation, i.e., the positions of " 1 "s in the $m^{\text {th }}$ row of the parity-check matrix. $N(m) \backslash l$ represents the set $L_{r}(m)$ with the $l^{\text {th }}$ bit excluded and $M(l) \backslash m$ represents the set $M(l)$ with the $m^{\text {th }}$ check excluded.

$q_{l \rightarrow m}^{i}$ Where $i=0,1$, denotes the probability information that the bit node $l$ sends to the check node $m$, indicated as $P\left(x_{l}=i\right)$.

While $r_{m \rightarrow l}^{i}$ denotes the probability information that the $m^{\text {th }}$ check node gathers for the $l^{\text {th }}$ bit being $i$. In other words $r_{m \rightarrow l}^{i}$ is the likelihood information for $x_{l}=i$ from the $m^{\text {th }}$ parity-check equation, when the probabilities for other bits are designated by the $q_{l \rightarrow m}^{i}$ so, $r_{m \rightarrow l}^{i}$ can be considered as the "extrinsic" information for the $l^{\text {th }}$ bit node from the $m^{\text {th }}$ check node. The "a posteriori probability" (APP) for a bit is calculated by gathering all the extrinsic information from the check nodes that connected to it. This can be obtained by the iterative belief propagation procedure. For binary codes, the sum-product algorithm can be performed more efficiently in Log domain, where the probabilities are equivalently characterized by the loglikelihood ratios $\quad$ (LLRs): $L\left(r_{m \rightarrow l}\right) \square \log \frac{r_{m \rightarrow l}^{1}}{r_{m \rightarrow l}^{0}}, \quad L\left(q_{m \rightarrow l}\right) \square \log \frac{q_{m \rightarrow l}^{1}}{q_{m \rightarrow l}^{0}}, \quad L\left(p_{l}\right) \square \log \frac{p_{l}^{1}}{p_{l}^{0}}$, $L\left(q_{l}\right) \square \log \frac{q_{l}^{1}}{q_{l}^{0}}$, note that $p_{l}^{i}$ represents the likelihood that the $l^{\text {th }}$ bit is $i$. The decoding algorithm (sum-product algorithm) steps will be explained [6]. 


\section{a. Initialization}

Each bit node $l$ is assigned an a priori LLR. $L\left(p_{l}\right)$. In case of equi-probable inputs on the channel with BPSK.

$$
L\left(p_{l}\right)=\log \frac{P\left(y_{l} \mid x_{l}=+1\right)}{P\left(y_{l} \mid x_{l}=-1\right)}
$$

where $x, y$ represent the transmitted bit and received bit respectively, for every position $(m, l)$ such that $\left(\mathbf{H}_{L D P C}\right)_{m l}=1$, where $\left(\mathbf{H}_{L D P C}\right)_{m l}$ represents the element of the $m^{\text {th }}$ row and the $l^{\text {th }}$ column in the parity-check matrix, $L\left(q_{l \rightarrow m}\right)$ and $L\left(r_{m \rightarrow l}\right)$ are initialized as:

$$
\begin{gathered}
L\left(q_{l \rightarrow m}\right)=L\left(P_{l}\right) \\
L\left(r_{m \rightarrow l}\right)=0
\end{gathered}
$$

\section{b. Checks to Bits}

Each check node $m$ gathers all the incoming information $L\left(q_{l \rightarrow m}\right)$ 's, and updates the belief on the bit $n$ based on the information from all other bits connected to the check node $m$.

$$
L\left(r_{m \rightarrow l}\right)=2 \tanh ^{-1}\left(\prod_{l^{\prime} \in L_{r}(m) \backslash l} \tanh \left(L\left(q_{l^{\prime} \rightarrow m}\right) / 2\right)\right)
$$

\section{c. Bits to checks}

Each bit node $n$ propagates its probability to all the check nodes that connect to it

$$
L\left(q_{l \rightarrow m}\right)=L\left(P_{l}\right)+\sum_{m \in M(l) \backslash m} L\left(r_{m^{\prime} \rightarrow l}\right)
$$

\section{d. Check Stop Criterion}

The decoder obtains the total a posteriori probability for the bit $l$ by summing the information from all the check nodes that connect to the bit $l$.

$$
L\left(q_{l}\right)=L\left(P_{l}\right)+\sum_{m \in M(l)} L\left(r_{m \rightarrow l}\right)
$$

Hard decision is made on the $L\left(q_{l}\right)$, and the resulting decoded input vector is checked against the parity-check matrix $\mathbf{H}_{L D P C}$. If $\mathbf{H}_{L D P C} \mathbf{y}=0$, the decoder stops and outputs input vector. Otherwise, it repeats the steps b, c and d until $\mathbf{H}_{L D P C} \mathbf{y}=0$, or a maximum number of iteration is set to stop the iteration.

\section{Serial Concatenation of LDPC Codes and RS Codes}

Concatenation of codes is a very useful technique, which leads to the construction of very efficient codes by using two or more constituent codes of relatively small size and complexity. There are essentially two ways of concatenating codes: traditionally, by using the so-called serial concatenation and more recently, by using the parallel concatenated structure of the first turbo coding schemes. Both concatenation techniques allow the use of iterative decoding [3], with a price of more delay time of the decoder processing, especially in serial concatenation.

In this work serial concatenation of codes is introduced between RS codes and LDPC codes a message block of $m K$ binary bits are first encoded by an outer Reed-Solomon $\left(N_{R S}, K\right)$ code, which generates a codeword of $m N_{R S}$ bits that are then encoded by the inner code $L D P C\left(N_{L D P C}, j, n\right)$, which generates a codeword of $N_{L D P C}$ bits. 


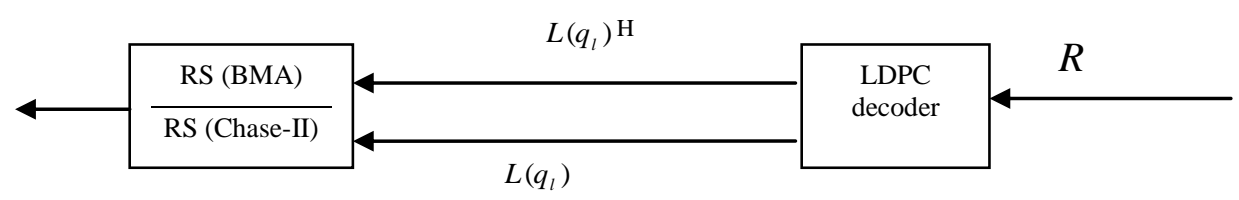

Fig. 3 Simple block diagram explain the concatenation of RS and GLDPC

Since delay is a major problem in serial concatenation, it was worth considering two decoding schemes in this work. The first scheme relays on a hard decision output of $L\left(q_{l}\right)$ from the inner LDPC code, to be used by Reed-Solomon codes in a Hard-In-Hard-Output fashion. The second scheme, shown in Fig. 3, will relay on soft $L\left(q_{l}\right)$ output from the inner LDPC code to be used by Reed-Solomon codes in a Soft-In-Hard-Output fashion. Comparing the performance of both schemes will be presented in the following section. These two schemes avoid the excess delay introduced in iterative decoding of serial concatenation since both codes

\section{Simulation Results}

In this section, the performance of the proposed serial concatenation schemes that uses RS $(63,55)$ as an outer code the regular $(6,3)$ LDPC as an inner code will be introduced. Simulation of both schemes will be carried out using an OFDM system over a mobile fading channel. The simulation foundation assumes the Rayleigh fading channel, the maximum delay spread is around $800 \mathrm{~ns}$, the minimum coherence bandwidth approximately equals $1.25 \mathrm{MHz}$, $N_{C}=64$, then the bandwidth of a single subcarrier become $78.125 \mathrm{kHz}$. Furthermore, with respect to the characteristics of the channel, if $x^{t}$ is chosen to consist of $N_{g}=6$ symbols, since the duration of the guard interval equals $1.2 \mathrm{~ms}$, which is larger than the maximum delay spread. We have chosen the guard interval to be greater than the maximum delay spread in order to avoid inter-symbol interference. These channel parameters match a LEO satellite channel model [16].

The output encoded data from RS-LDPC will be of size 756 bits, where an outer RS $(63,55)$ over GF $\left(2^{6}\right)$, and an inner LDPC $(756,2,15)$ code are used. The overall code rate is 0.4666 . The binary equivalent Reed-Solomon encoder accepts 55x6=330 bits as input and outputs $63 \times 6=378$ binary bits. The inner LDPC encoder uses a parity check matrix of size $378 \times 756$ which results in an encoded bit stream of length 756 bits.

This transmitted vector is transmitted using OFDM modulation over the used channel and after reception the noisy received 756 bit stream will enter the LDPC decoder. The LDPC decoder uses the Sum-Product Algorithm [8] which can output either a soft or hard decision values. The number of iteration for both decoding scheme is set to 4 .

In the first decoding scheme Reed-Solomon decoder is a Hard-Input-Hard-Output that accepts 378 bits and it uses Berlekamp-Massey Algorithm to decode them into 330 recovered received bits. In the second decoding scheme Reed-Solomon works in Soft-Input-HardOutput mode. It uses the Chase-II algorithm with number of least reliable bits equals 3 that results in 8 test error patterns. Although this number can be increased; but it will be associated with exponential complexity. 


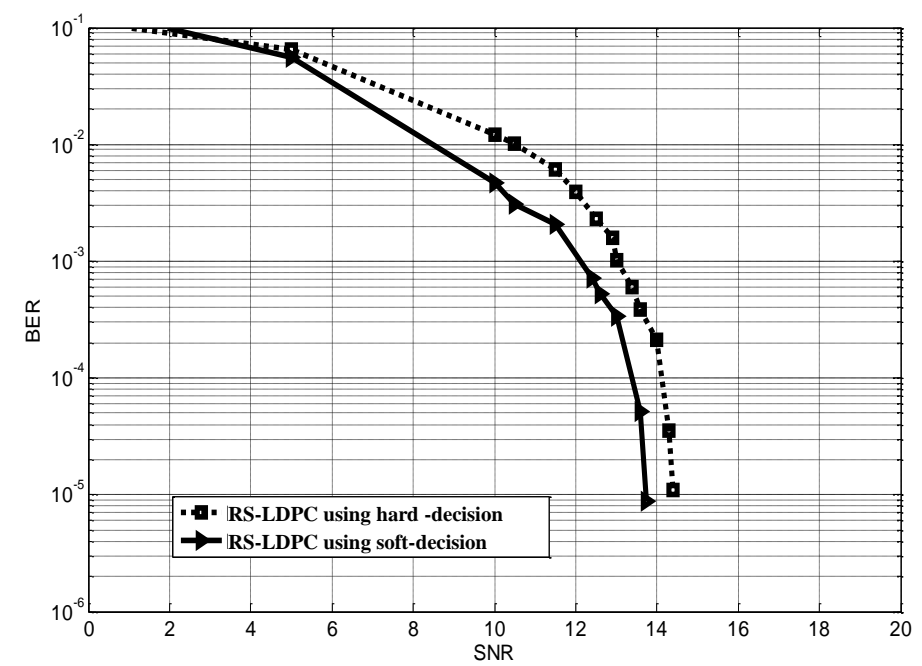

Fig. 6 Comparison between two decoding schemes

Figure 6 shows the BER performance of the two decoding schemes using OFDM modulation over a Raleigh fading channel. It is clear that soft decision decoding, even with a limited number of test error patterns, gives significant improvement over hard decision decoding of Reed-Solomon Codes. At BER $=10^{-5}$, soft decision decoding can achieve a coding gain of 1-dB. Although more coding gain can be obtained by either increasing the soft-decision decoding capability of Chase-II algorithm or increase the number of iteration of LDPC decoder, but one has to consider the added complexity to the system.

\section{Conclusions}

LDPC codes are attractive channel codes especially for OFDM. Concatenation with LDPC can improve the performance of these systems. Because of their superior performance in correcting burst errors, Reed Solomon codes have been concatenated with LDPC codes. In this work a serial concatenation, which is far simpler to implement than parallel concatenation, between an outer Reed Solomon code and an inner LDPC code has been presented. Two decoding schemes have been investigated for decoding Reed-Solomon codes. Soft-decision decoding of Reed Solomon code using Chase-II algorithm has resulted in 1-dB coding gain compared with the first decoding scheme based on hard-decision decoding. Further coding gain of the second decoding scheme is possible either by increasing the number of test error patterns of Chase-II algorithm or by increasing the number of decoding iterations of the LDPC code. However, one must consider the excess produced delay due to the nature of serial concatenation.

\section{References}

[1] R. Gallager, "Low density parity check codes," IRE Trans. on Inform. Theory, vol. IT-8, pp. 21-28, Jan. 1962.

[2] D. MacKay, "Good error-correcting codes based on very sparse matrices," IEEE Trans. on Inform. Theory, vol. 45, pp. 399-431, Mar. 1999.

[3] A. G. C. Berrou, et al., "Near-Optimum error correction coding and decoding: Turbo codes," IEEE Transaction on Communications, vol. 44, pp. 1261-1271, 1996.

[4] R. M. Pyndiah, "Near-Optimum Decoding of product codes: Block Turbo Codes," IEEE Transaction on Communications, vol. 46, pp. 1003-1010, 1998. 
[5] Yun Kim, Iickho Song "Performance analysis of a coded OFDM system in time-varying multipath Rayleigh fading channels," IEEE Trans. on Vehicular Technology, vol. 48, no. 5, pp. 1610-1615, Sept. 1999.

[6] C.Yuan, Y.Mong-Kai "LDPC coded OFDM modulation for high spectral efficiency transmission," Proceedings of the 4th European Conference on Circuits and Systems for Communications, 10-11 July, 2008, pp. 280-284.

[7] D. Abematsu, , T. Ohtsuki, and T. Kashima, and S. Jarot, "LDPC codes for high data rate multiband OFDM systems over 1Gbps," Proceedings of the IEEE Pacific Rim Conference on Communications, Computers and Signal Processing, 22-24 Aug., 2007, pp. 338-341.

[8] Ali Serener "Performance of spread OFDM with LDPC coding in outdoor," Proceedings of the $58^{\text {th }}$ IEEE Vehicular Technology Conference, 6-9 Oct., 2003, pp. $318-32$.

[9] S. Basharat, Y. Rao, "Design and performance of LDPC codes for OFDM based aeronautical communication systems" Proceedings of the $3^{\text {rd }}$ Advanced International Conference on Telecommunications, 13-19 May, 2007, pp. 24-24.

[10] Djurdjevic, J. Xu, K. Abdel-Ghaffar and S. Lin,"A class of low-density parity-check codes constructed based on Reed-Solomon codes with two information symbols," IEEE Communications Letters, vol. 7, no. 7, pp. 317-319, July 2003.

[11] Chao Chen; Baoming Bai; Xinmei Wang, "Construction of quasi-cyclic LDPC codes based on a two-dimensional MDS code," IEEE Communications Letters, vol.14, no.5, pp.447-449, May 2010.

[12] Z. Zhang, V. Anantharam, M. J. Wainwright, B. Nikolic,"An efficient 10GBASE-T Ethernet LDPC decoder design with low error floors," IEEE Journal of Solid-State Circuits, vol. 56, no. 4, pp. 843-855, April 2010.

[13] Hwang, Seong-In ; Hanho Lee ; Lim, Shin-Il, "A novel method of constructing QuasiCyclic RS-LDPC codes for 10GBASE-T Ethernet, IEEE International Symposium on Circuits and Systems (ISCAS) 20-22 May, 2012, pp. 1771-1774.

[14] S.B. Wicker, Reed-Solomon codes and their applications. Piscataway, NJ: IEEE Press, 1994.

[15] D. Chase, "Class of algorithms for decoding block codes with channel measurement information," IEEE Transactions on Information Theory, vol. 18, pp. 170-182, 1972.

[16] A. Papathanassiou, A. Salkintzis, and P. Mathiopoulos "A comparison study of the uplink performance of W-CDMA and OFDM for mobile multimedia communications via LEO satellites" IEEE Trans. on Personal Communications. vol. 8, no. 3, pp. 35-43, June 2001. 